www.jmscr.igmpublication.org

Impact Factor 5.84

Index Copernicus Value: 83.27

ISSN (e)-2347-176x ISSN (p) 2455-0450

crossref DOI: _https://dx.doi.org/10.18535/jmscr/v5i7.226

\title{
Immunoglobulin G4-Related Disease (IgG4-RD) Presenting as Sclerosing Cholangitis - A Radiological Case Report
}

\author{
Authors \\ Kirti Rana Chaturvedy ${ }^{1}$, Navjeet Kaur ${ }^{2}$, Ramanand Gehlot ${ }^{3}$, Preeti Meena ${ }^{4}$ \\ ${ }_{1,3}^{1,3}$ Professor, Department of Radiodiagnosis \\ ${ }^{2,4}$ Resident, Department of Radiodiagnosis \\ Dr. Sampurnanand Medical College, Jodhpur, Rajasthan 342003 \\ Corresponding Author
}

Dr Navjeet Kaur

Resident, Dept of Radiodiagnosis, Dr.Sampurnanand Medical College, Jodhpur, Rajasthan 342003

Email: navjeetsamra@gmail.com

\begin{abstract}
Immunoglobulin G4-related disease (IgG4-RD) is a rare systemic fibro-inflammatory disorder mimicking inflammatory and neoplastic processes. Numerous extrapancreatic organs such as the bile ducts, gallbladder, kidneys, retroperitoneum, thyroid, salivary glands, lung, mediastinum, lymph nodes, and prostate may be involved, either synchronously or metachronously. This case report of a 36 year old Indian male aims to highlight the imaging spectrum of multi-organ involvement in a case of IgG4 related disease. It is important to recognize multiorgan involvement of IgG4-related sclerosing disease and be familiar with its clinical and imaging features because it demonstrates a favourable response to treatment. Our case report leads us to reflect on the necessity to take into account this disease in patients with multisystem involvement. Keywords: Immunoglobulin G4 related disease, sclerosing cholangitis, phlebitis, thrombosis, fibrosclerosing lesion.
\end{abstract}

\section{INTRODUCTION}

Immunoglobulin G4- related disease (IgG4-RD) is a recently defined emerging systemic fibroinflammatory disorder of unknown origin, characterized by a diffuse or mass forming inflammatory reaction rich in IgG4-positive plasma cells associated with fibrosclerosis and obliterative phlebitis.

The IgG4-related disease has been described in several organs and tissues: pancreas, biliary tract, salivary glands, periorbital tissue, kidneys, lungs, lymph nodes, meninges, aorta, breast, prostate, thyroid, pericardium, and skin. ${ }^{[1]-[3]}$
Elevated IgG4 serum concentrations are noticed in 60-70\% of patients.

Responsiveness to glucocorticoids is reported, particularly in early stages of the disease.

This case report aims to highlight the imaging spectrum of multi-organ involvement in a case of IgG4 related disease.

\section{CASE REPORT}

A 36 year old Indian male presented with one month history of gradual onset of bilateral leg swelling, difficulty in walking, right hypochondrial pain and jaundice. 


\section{JMSCR Vol||05||Issue||07||Page 25686-25689||July}

Clinical examination revealed icterus, bilateral pedal oedma and left lower limb spasticity. Right hypochondrial lump was palpable per abdomen. Liver function tests were deranged with elevated enzymes and direct bilirubin.

No markers to suggest pancreatic inflammation were found.

Ultrasonography showed inferior vena caval thrombosis. Portal vein thrombosis with features of portal hypertension were seen. Mild splenomegaly was present. Multiple peri-splenic and mesenteric collaterals were seen (Fig. 1).

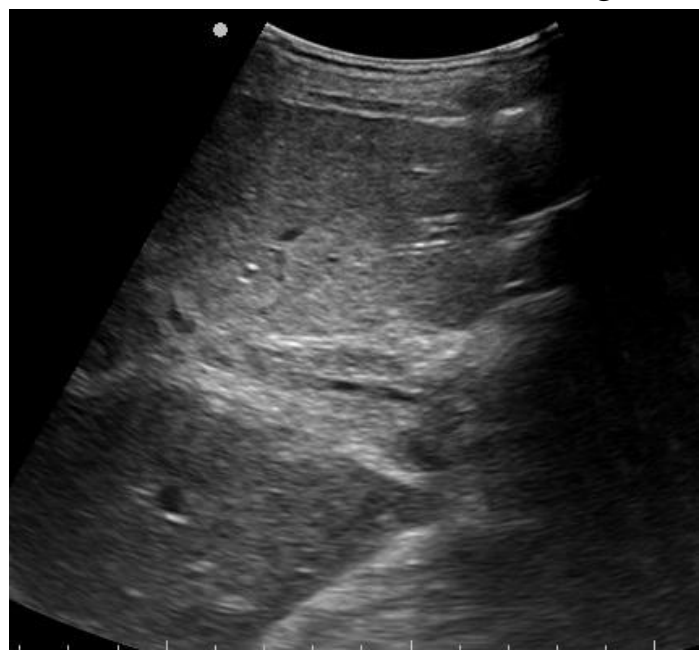

(a)

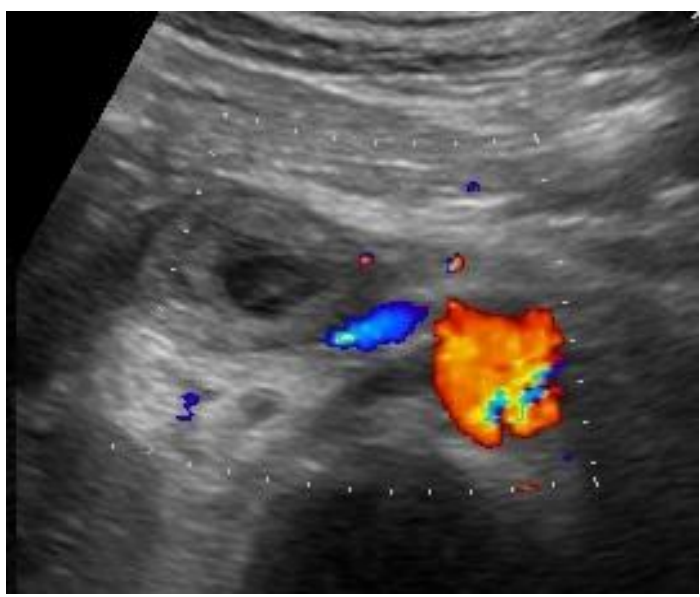

(b)

Fig. 1 Ultrasound images showing periportal soft tissue thickening (a) and IVC thrombosis (b) Contrast enhanced computed tomography (CECT) of abdomen showed mildly bulky pancreatic head. Soft tissue proliferation was seen at porta hepatis and around extrahepatic biliary tree. Portal and splenic veins were thrombosed. Plaque-like soft tissue thickening was noted in para-aortic, para- caval, perinephric regions and encasing the right PUJ, typical of retroperitoneal fibrosis. Infrahepatic inferior vena cava and its tributaries were thick walled, dilated and thrombosed. Right renal vein was also thrombosed. Hepatic IVC and hepatic veins were normal (Fig. 2).

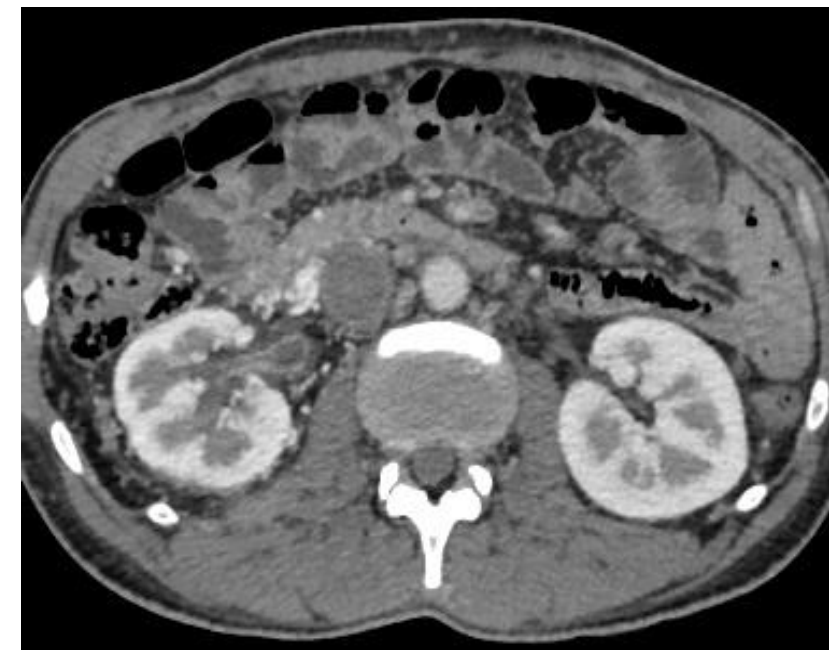

(a)

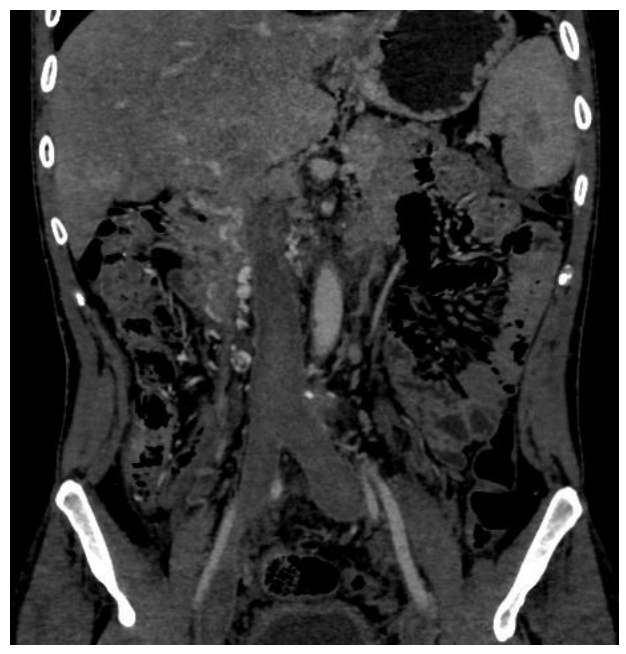

(b)

Fig. 2 CECT images showing soft tissue density around right PUJ (Retroperitoneal fibrosis) (a) and IVC thrombosis (b)

A presumptive diagnosis of $\mathrm{IgG} 4$ related disease was made and patient was further referred for laboratory confirmation and magnetic resonance cholangio-pancreatography (MRCP).

Serum IgG4 levels $(4.01 \mathrm{~g} / \mathrm{l})$ were significantly elevated (normal 0.03-2.01g/l). Serum antinuclear antibodies were mildly elevated (2+).

Multiple randomly distributed areas of smooth narrowing and focal dilatations were seen in left bile duct (beading) and uniform narrowing in right 
branch with poor visualisation of peripheral IHBRs (Fig. 3).

The patient was treated with corticosteroids and anticoagulants which resulted in a reduction in pedal oedema and jaundice.

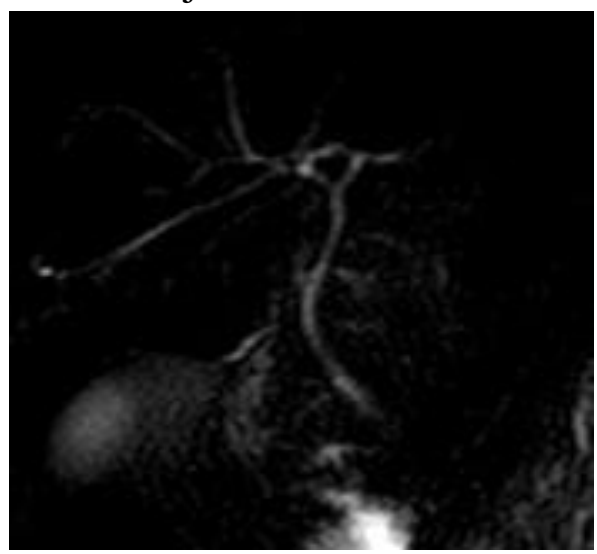

Fig. 3 MRCP single shot images show beading of left bile duct and IHBRs

Follow up scan after four months revealed mild resolution of retroperitoneal soft tissue thickening.
IVC was reduced in calibre with streaky luminal flow.

\section{DISCUSSION}

IgG4-related sclerosing cholangitis is the most frequent extrapancreatic manifestation of $\mathrm{IgG} 4$ RD. It also rarely occurs in the absence of pancreatitis. Biliary tract involvement is seen in $50 \%$ to $90 \%$ of patients with IgG4-related sclerosing pancreatitis and clinically presents as obstructive jaundice or fever. ${ }^{[4]-[6]}$

Retroperitoneal fibrosis is one of the most commonly encountered subsets of IgG4-RD. Inferior vena cava thrombosis is a rare manifestation of this disorder and is considered secondary to compression by retroperitoneal fibroinflammatory tissue.

Table 1- Asian Diagnostic Criteria for Autoimmune Pancreatitis (IgG4-realted Sclerosing Pancreatitis)

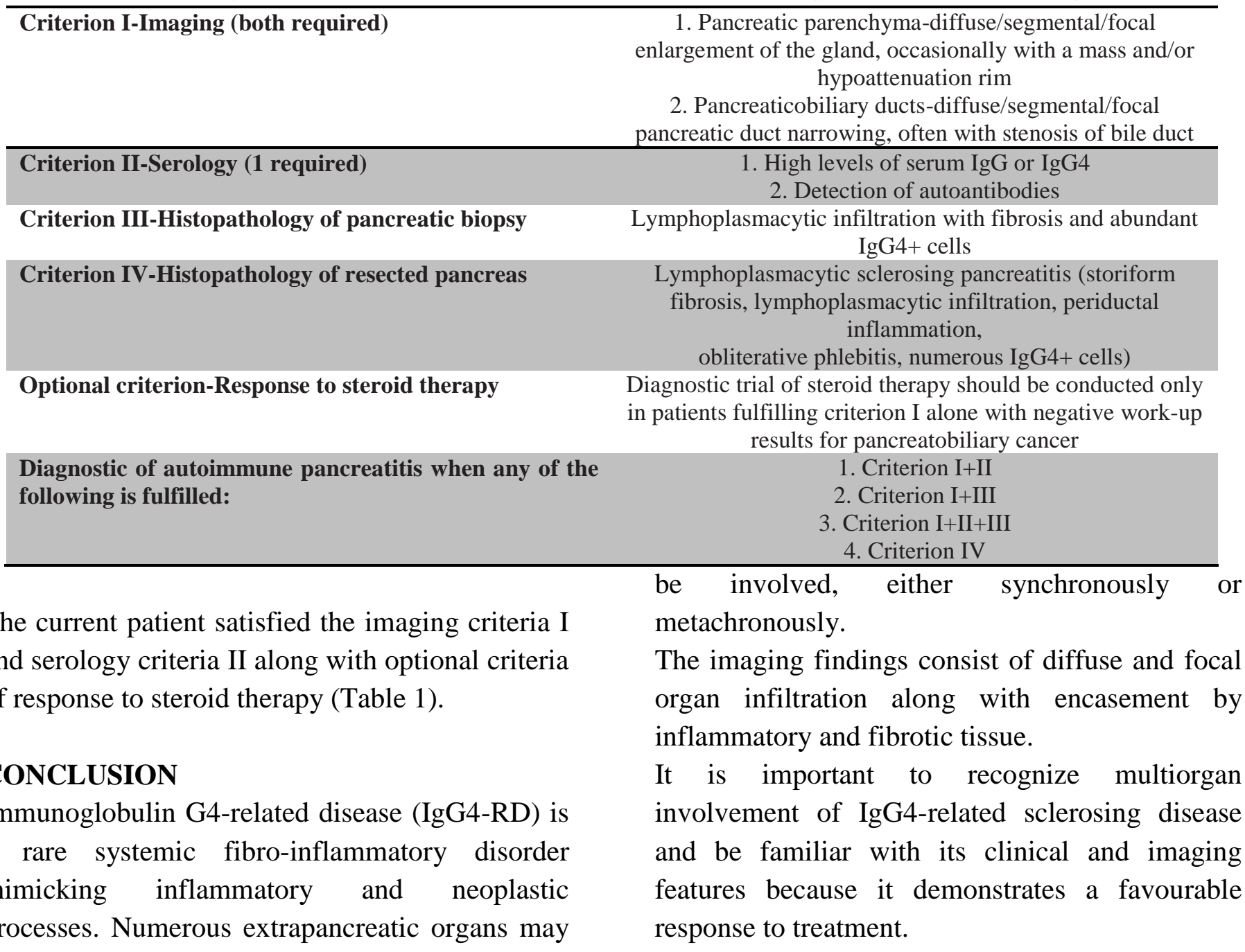


Our case report leads us to reflect on the necessity to take into account this disease in patients with multisystem involvement especially in patients with concomitant occurrence of retroperitoneal fibrosis and sclerosing cholangitis.

This case report is unique as the patient presented primarily with extensive venous obliterative phlebitis and thrombosis without significant laboratory evidence of pancreatic inflammation.

Conflicts of interest- Nil

\section{REFERENCES}

1. J. H. Stone, Y. Zen, and V. Deshpande, "IgG4-related disease," The New England Journal of Medicine, vol. 366, no. 6, pp. 539-551, 2012.

2. W. Cheuk and J. K. C. Chan, "IgG4related sclerosing disease: a critical appraisal of an evolving clinicopathologic entity," Advances in Anatomic Pathology, vol. 17, no. 5, pp. 303-332, 2010.

3. Khosroshahi and J. H. Stone, "A clinical overview of IgG4-related systemic disease," Current Opinion in Rheumatology, vol.23, no. 1, pp. 57-66, 2011.

4. Kawaguchi K, Koike M, Tsuruta K, Okamoto A, Tabata I, Fujita N. Lymphoplasmacytic sclerosing pancreatitis with cholangitis:a variant of primary sclerosing cholangitis extensively involving pancreas. Hum Pathol1991;22:387-95

5. Zen Y, Harada K, Sasaki M, Sato Y, Tsuneyama K, Haratake J, et al. IgG4related sclerosing cholangitis with and without hepatic inflammatory pseudotumor, and sclerosing pancreatitisassociated sclerosing cholangitis: do they belong to a spectrum of sclerosing pancreatitis? Am J Surg Pathol 2004;28: 1193-203.

6. Deshpande V, Sainani NI, Chung RT, Pratt DS, Mentha G, Rubbia-Brandt L, et al. IgG4-associated cholangitis: a comparative histological and immunophenotypic study with primary sclerosing cholangitis on liver biopsy material. Mod Pathol 2009;22:1287-95. 